\title{
Inclusive and diffractive $W$ boson production asymmetry at the LHC
}

\section{K. Golec-Biernat ${ }^{* \dagger}$}

Institute of Nuclear Physics PAN, Kraków, Poland

Institute of Physics, Rzeszow University, Rzeszów, Poland

E-mail: golec@ifj.edu.pl

\section{A. Łuszczak}

Institute of Nuclear Physics PAN, Kraków, Poland

E-mail: Agnieszka.Luszczak@ifj.edu.pl

\begin{abstract}
We analyse diffractive electroweak vector boson production at the LHC and show that the single diffractive $W$ boson production asymmetry in rapidity is a particularly good observable to test the concept of the flavour symmetric pomeron parton distributions. It may also provide an additional constraint for parton distribution functions in the proton.
\end{abstract}

XVIII International Workshop on Deep-Inelastic Scattering and Related Subjects, DIS 2010 April 19-23, 2010

Firenze, Italy

\footnotetext{
* Speaker.

†Presentation based on Ref. [1].
} 


\section{Introduction}

The electroweak $W$ and $Z$ boson production in hadronic collision is a particularly valuable process to constrain parton distribution functions (PDFs) in a nucleon. By measuring leptonic products of the weak boson decays, electroweak parameters like $\sin ^{2} \theta_{W}$ or the $W / Z$ boson masses and decay widths can also be determined. At the Born level, the $W$ and $Z$ bosons are produced by annihilation of two quarks from the colliding nucleons. In the collinear approximation, the elementary cross sections for these processes have to be convoluted with the nucleons' PDFs. A direct access to these distributions is provided by the measurement of $W^{ \pm}$production asymmetry in rapidity. The measured $W$ asymmetry can be used in the global fit analysis to constrain PDFs, in particular, the ratio of the $u$ and $d$ PDFs. Such measurements were performed at the Fermilab Tevatron $[2,3,4,5,6]$.

Diffractive hadroproduction of electroweak bosons was observed experimentally at the Tevatron [7] and analysed theoretically in a series of papers [8, 9, 10, 11]. In the pomeron model interpretation, the rapidity gap between the intact hadron and the diffractive system appears due to a vacuum quantum number exchange with partonic substructure, in the case when a hard scale exists in the process. [12]. This structure is described by the pomeron parton distributions which are usually assumed to be flavour symmetric to account for vacuum quantum numbers of the pomeron exchange. We will show that the measurement of the diffractive $W$ boson asymmetry at the LHC is an ideal process to test the latter assumption. In addition, this asymmetry may provide an additional constraint for the determination of the ordinary PDFs in the proton.

\section{Inclusive case}

The leading order cross sections for the $W$ bosons production are given by [13]

$$
\frac{d \sigma_{W^{ \pm}}}{d y}=\sigma_{0}^{W} \sum_{q q^{\prime}}\left|V_{q q^{\prime}}\right|^{2}\left\{q\left(x_{1}, \mu\right) \bar{q}^{\prime}\left(x_{2}, \mu\right)+\bar{q}\left(x_{1}, \mu\right) q^{\prime}\left(x_{2}, \mu\right)\right\}
$$

where the factorization scale $\mu=M_{W}$, and $q / \bar{q}$ denote quark/antiquark distributions. In addition

$$
\sigma_{0}^{W}=\frac{2 \pi G_{F}}{3 \sqrt{2}} \frac{M_{W}^{2}}{s}, \quad x_{1}=\frac{M_{W}}{\sqrt{s}} \mathrm{e}^{y}, \quad x_{2}=\frac{M_{W}}{\sqrt{s}} \mathrm{e}^{-y}
$$

where $y$ is the $\mathrm{W}$ boson rapidity. Obviously, $y=\frac{1}{2} \ln \left(x_{1} / x_{2}\right)$ and from the conditions $0<x_{1,2}<1$, the following constraint results: $-y_{\max }<y<y_{\max }$ with $y_{\max }=\ln \left(\sqrt{s} / M_{W}\right)$. The cross section for the $Z$ boson production has a similar form. In the forthcoming analysis we consider only $u$ and $d$ quarks since the $s$ quark is Cabbibo suppressed. The $W^{ \pm}$boson production asymmetry in rapidity is defined as follows

$$
A(y)=\frac{d \sigma_{W^{+}}(y) / d y-d \sigma_{W^{-}}(y) / d y}{d \sigma_{W^{+}}(y) / d y+d \sigma_{W^{-}}(y) / d y} .
$$

Substituting relavant quark distributions, we find

$$
A(y)=\frac{\left(u_{p}\left(x_{1}\right)-d_{p}\left(x_{1}\right)\right) \bar{u}_{p}\left(x_{2}\right)+\bar{u}_{p}\left(x_{1}\right)\left(u_{p}\left(x_{2}\right)-d_{p}\left(x_{2}\right)\right)}{\left(u_{p}\left(x_{1}\right)+d_{p}\left(x_{1}\right)\right) \bar{u}_{p}\left(x_{2}\right)+\bar{u}_{p}\left(x_{1}\right)\left(u_{p}\left(x_{2}\right)+d_{p}\left(x_{2}\right)\right)},
$$


W and $\mathrm{Z}$ production cross sections at LHC

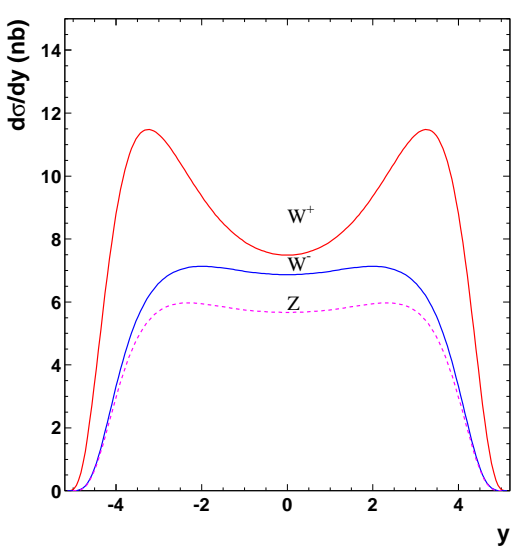

W asymmetry at LHC

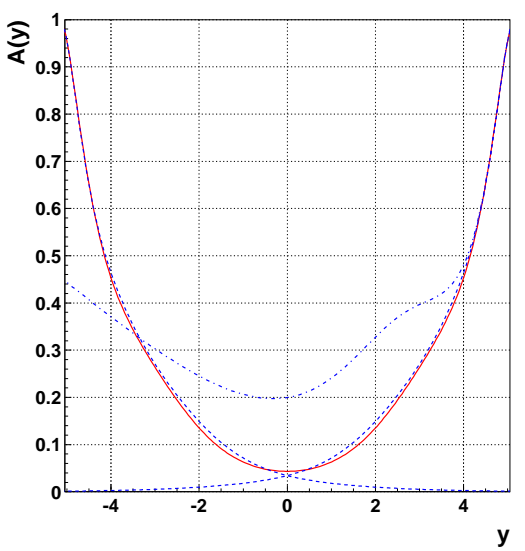

Figure 1: $\mathrm{T}$ The $W$ and $Z$ boson production cross sections at the LHC and asymmetry $A(y)$ computed for the LO MSTW08 parton distributions [14]. The dashed lines on the right show relation (2.5) while the dash-dotted line show the approximation with valence quarks only.

where the local isospin symmetry for the sea quarks, $\bar{u}_{p}(x)=\bar{d}_{p}(x)$, was assumed for simplicity. The asymmetry is symmetric with respect to the rapidity reflection $y \rightarrow-y$, which is clearly seen in Fig. 1. For $x_{1} \sim 1$, the sea quark distribution $\bar{u}\left(x_{1}\right)$ is small and

$$
A(y) \simeq \frac{u_{p}\left(x_{1}\right)-d_{p}\left(x_{1}\right)}{u_{p}\left(x_{1}\right)+d_{p}\left(x_{1}\right)} .
$$

When $x_{2} \sim 1$ the argument in the above is changed to $x_{2}$. From this relation, the $d_{p}(x) / u_{p}(x)$ ratio at the scale $\mu=M_{W}$ can easily be found.

\section{Diffractive case}

In the single diffractive case, the electroweak bosons are produced in a restricted region of rapidity with a rapidity gap without particles between the proton, which stayes intact, and the diffractive system. In the pomeron model interpretation, one of two quarks which produce the weak bosons, comes form the pomeron and is described by flavour symmetric pomeron parton distributions

$$
u_{\mathbb{P}}=\bar{u}_{\mathbb{P}}=d_{\mathbb{P}}=\bar{d}_{\mathbb{P}}=s_{\mathbb{P}}=\bar{s}_{\mathbb{P}}=\ldots \equiv q_{\mathbb{P}}\left(x, x_{\mathbb{P}}\right),
$$

where $x_{I P}=M_{D}^{2} / s$ is a fraction of the proton's momentum transferred into the diffractive system of mass $M_{D}$ and $x=x_{2} / x_{I P}$ is a fraction of the pomeron momentum carried by the pomeron parton. From the conditions $0<x, x_{\mathbb{P}}<1$, the $W$ boson rapidity is in the range: $-y_{\max }+\ln \left(1 / x_{\mathbb{P}}\right)<y<$ $y_{\max }$. Thus $\Delta=\ln \left(1 / x_{\mathbb{P}}\right)$ is the length of the rapidity gap .

In Fig. 2 we show the $W$ and $Z$ production cross sections with the LO MSTW08 proton parton distributions [14] and the pomeron parton distributions from the analysis [15]. The effect of the pomeron is clearly visible in the left hemisphere - the rapididty gap is formed and the $W$ asymmetry strongly decreases. These cross sections should be multiplied by a gap survival factor, $S^{2}=0.09$ [16], which takes into account soft interactions destroying the rapidity gap [17]. 

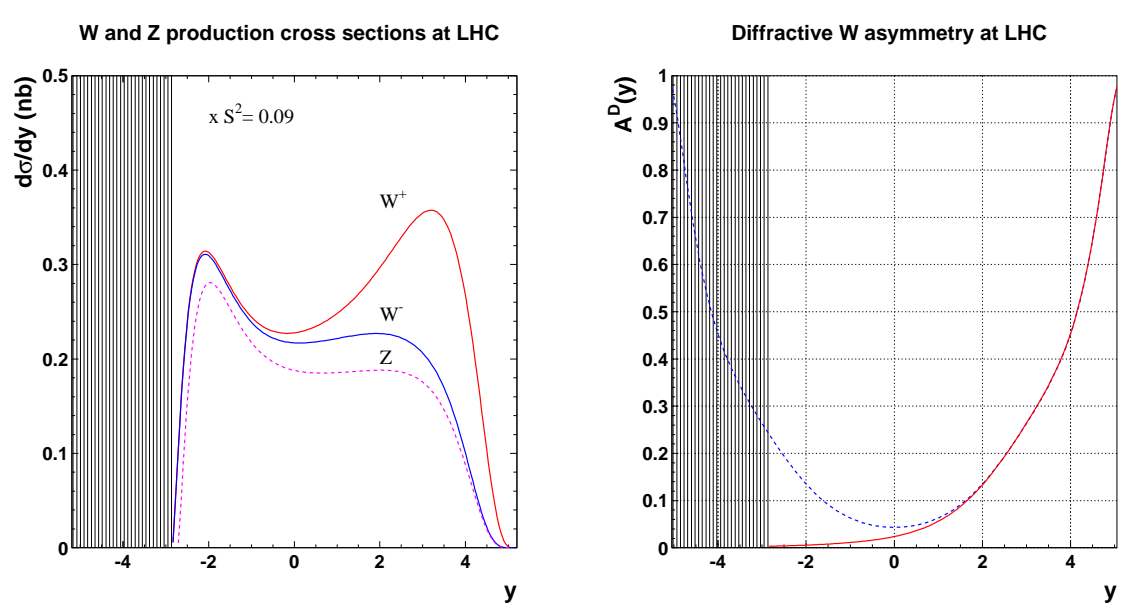

Figure 2: The diffractive weak boson production cross sections and the corresponding $W$ boson asymmetry (solid line) together with asymmetry in the inclusive case (dashed line). The cross sections have to be multiplied by the gap survival factor $S^{2}=0.09$. The shaded area indicate the rapidity gap $\Delta\left(x_{\mathbb{P}}=0.1\right)=2.3$.

The $W$ boson production asymmetry (2.3) is a particularly good observable since it is insensitive to the gap survival factor, which multiplies the diffractive $W$ productions cross sections. The flavour symmetric pomeron parton distributions also cancel, and we obtain for the $W$ boson asymmetry in the diffractive case,

$$
A^{D}(y)=\frac{u_{p}\left(x_{1}\right)-d_{p}\left(x_{1}\right)}{u_{p}\left(x_{1}\right)+d_{p}\left(x_{1}\right)+2 \bar{u}_{p}\left(x_{1}\right)} .
$$

Notice that $A^{D}(y)$ is independent of $x_{\mathbb{P}}$, i.e. the length of the rapidity gap. In order to understand our result, it is interesting to compare Eq. (3.2) with the approximate asymmetry (2.5), valid in the right hemisphere for $y>0$. For large rapidities, when the sea quark distributions can be neglected, these two asymmetries are equal while for $y \approx 0$, when the valence quark distributions in the denominator are negligible, $A^{D}(y) \approx A(y) / 2$. This is clearly seen in Fig. 3 where the ratio $A^{D}(y) / A(y)$ is shown. Approaching the rapidity gap, the asymmetry $A^{D}(y) \rightarrow 0$ while $A(y)$ rises. Thus, the ratio shown in Fig. 3 is close to zero at the edge of the rapidity gap.

The pattern shown in Fig. 3 is quite general and depends only on the assumption on flavour symmetry of the pomeron parton distributions. Therefore, it would be interesting to test experimentally the very concept of the flavour symmetric pomeron parton distributions by measuring the ratio of the two $W$ asymmetries in the diffractive and nondiffractive $p p$ scattering. Systematic errors will cancel in such a ratio which should allow for a quite precise determination of this quantity. In addition, the diffractive $W$ asymmetry provides an additional constraint for the parton distribution functions in the proton. We are looking forward to the experimental verification at the LHC of the presented results.

\section{Acknowledgments}

This work is partially supported by the grants of MNiSW Nos. N N202 246635 and N N202 


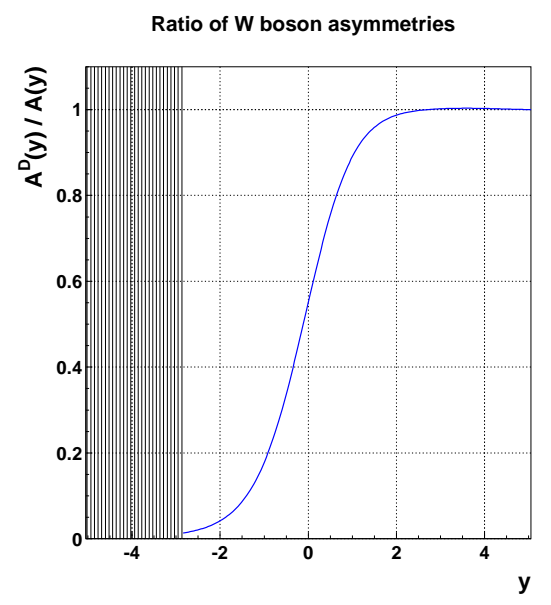

Figure 3: The ratio of the $W$ asymmetries in the diffractive and nondiffractive $p p$ scattering.

249235, and the grant HEPTOOLS, MRTN-CT-2006-035505.

\section{References}

[1] K. Golec-Biernat and A. Luszczak, Phys. Rev. D81, 014009 (2010), [0911.2789].

[2] CDF, F. Abe et al., Phys. Rev. Lett. 74, 850 (1995), [hep-ex/9501008].

[3] D0, V. M. Abazov et al., Phys. Rev. Lett. 101, 211801 (2008), [0807.3367].

[4] CDF, T. Aaltonen et al., Phys. Rev. Lett. 102, 181801 (2009), [0901.2169].

[5] CDF, F. Abe et al., Phys. Rev. Lett. 77, 2616 (1996).

[6] D0, V. M. Abazov et al., Phys. Rev. Lett. 101, 191801 (2008), [0804.3220].

[7] CDF, F. Abe et al., Phys. Rev. Lett. 78, 2698 (1997), [hep-ex/9703010].

[8] R. J. M. Covolan and M. S. Soares, Phys. Rev. D60, 054005 (1999), [hep-ph/9905352].

[9] R. J. M. Covolan and M. S. Soares, Phys. Rev. D67, 017503 (2003), [hep-ph/0212349].

[10] R. J. M. Covolan and M. S. Soares, Phys. Rev. D67, 077504 (2003), [hep-ph/0305186].

[11] M. B. Gay Ducati, M. M. Machado and M. V. T. Machado, Phys. Rev. D75, 114013 (2007), [hep-ph/0703315].

[12] G. Ingelman and P. E. Schlein, Phys. Lett. B152, 256 (1985).

[13] R. K. Ellis, W. J. Stirling and W. B. R., QCD and Collider Physics, Cambridge University Press (1996).

[14] A. D. Martin, W. J. Stirling, R. S. Thorne and G. Watt, 0901.0002.

[15] K. J. Golec-Biernat and A. Luszczak, Phys. Rev. D76, 114014 (2007), [0704.1608].

[16] V. A. Khoze, A. D. Martin and M. G. Ryskin, Eur. Phys. J. C18, 167 (2000), [hep-ph/0007359].

[17] J. D. Bjorken, Phys. Rev. D47, 101 (1993). 